\title{
Horizontal branch A- and B-type stars in globular clusters
}

\author{
Sabine Möhler \\ Institut für theoretische Physik und Astrophysik, Christian-Albrechts-Universität zu Kiel, \\ Olshausenstraße 40, D 24118 Kiel, Germany \\ email: moehler@astrophysik.uni-kiel.de
}

\begin{abstract}
Globular clusters offer ideal laboratories to test the predictions of stellar evolution. When doing so with spectroscopic analyses during the 1990s, however, the parameters we derived for hot horizontal branch stars deviated systematically from theoretical predictions. The parameters of cooler, A-type horizontal branch stars, on the other hand, were consistent with evolutionary theories. In 1999, two groups independently suggested that diffusion effects might cause these deviations, which we verified subsequently. I will discuss these observations and analyses and their consequences for interpreting observations of hot horizontal branch stars.
\end{abstract}

Keywords. Diffusion, stars: atmospheres, stars: evolution, stars: horizontal branch, Galaxy: globular clusters: general

\section{Historical background}

Globular clusters are densely packed, gravitationally bound systems of several thousand to about one million stars. The dimensions of the globular clusters are small compared to their distance from us. Half of the light is generally emitted within a radius of less than $10 \mathrm{pc}$, whereas the closest globular cluster has a distance of $2 \mathrm{kpc}$ and $90 \%$ lie more than $5 \mathrm{kpc}$ away. We can thus safely assume that all stars within a globular cluster lie at the same distance from us. With ages in the order of $10^{10}$ years globular clusters are among the oldest objects in our Galaxy. As they formed stars only once in the beginning and the duration of that star formation episode is short compared to the current age of the globular clusters, the stars within one globular cluster are essentially coeval. In addition all stars within one globular cluster (with few exceptions) show the same initial abundance pattern (which may differ from one cluster to another). Globular clusters are thus the closest approximation to a physicists' laboratory in astronomy.

The horizontal branch, which is the topic of this article, was discovered by ten Bruggencate (1927), when he used Shapley's (1915) data on M 3 and other clusters to plot magnitude versus colour (replacing luminosity and spectral type in the HertzsprungRussell diagram) and thus produced the first colour-magnitude diagrams ("FARBENHELLIGKEITSDIAGRAMME"). In these colour-magnitude diagrams (CMDs) ten Bruggencate noted the presence of a red giant branch that became bluer towards fainter magnitudes, in agreement with Shapley (1915). In addition, however, he saw a horizontal branch ("HORIZONTALER AST") that parted from the red giant branch and extended far to the blue at constant brightness. As more CMDs of globular clusters were obtained it became obvious that the relative numbers of red and blue horizontal branch stars (i.e., the horizontal branch morphology) varied quite considerably between individual clusters, with some clusters showing extensions of the blue horizontal branch (so-called "blue tails") towards bluer colours and fainter visual magnitudes, i.e., towards hotter temperaturest.

$\dagger$ The change in slope of the horizontal branch towards higher temperatures is caused by 
Sandage \& Wallerstein (1960) noted a correlation between the metal abundance and the horizontal branch morphology seen in globular cluster CMDs, the horizontal branch (HB) became bluer with decreasing metallicity.

About 25 years after the discovery of the horizontal branch Hoyle \& Schwarzschild (1955) were the first to identify horizontal branch stars with post-red giant branch stars that burn helium in the central regions of their cores. Faulkner (1966) managed for the first time to compute zero age horizontal branch (ZAHB) models that qualitatively reproduced the observed trend of $\mathrm{HB}$ morphology with metallicity without taking into account any mass loss but assuming a rather high helium abundance of $Y=0.35$. Iben \& Rood (1970), however, found that they could "... account for the observed spread in colour along the horizontal branch by accepting that there is also a spread in stellar mass along this branch, bluer stars being less massive (on the average) and less luminous than redder stars." Comparing HB models to observed globular cluster CMDs Rood (1973) found that an HB that "... is made up of stars with the same core mass and slightly varying total mass, produces theoretical $c-m$ diagrams very similar to those observed. .... A mass loss of perhaps $0.2 M_{\odot}$ with a random dispersion of several hundredths of a solar mass is required somewhere along the giant branch." The assumption of mass loss on the red giant branch diminished the need for very high helium abundances.

Thus our current understanding sees horizontal branch stars as stars that burn helium in a core of about $0.5 M_{\odot}$ and hydrogen in a shell and evolve to the asymptotic giant branch, when the helium in the core is exhausted (for a review on HB evolution see Sweigart 1994). The more massive the hydrogen envelope e.g. the cooler is the resulting star at a given metallicity $\dagger$. The masses of the hydrogen envelopes vary from $\leqslant 0.02 M_{\odot}$ at the hot end of the horizontal branch (about $30000 \mathrm{~K}$ ) to $0.3-0.4 M_{\odot}$ for the cool HB stars at about 4000-5000 K (depending on metallicity, e.g., Dorman et al. 1993). The stable red and blue $\mathrm{HB}$ stars are separated by the variable RR Lyrae range at about 6500-7500 K. This article deals with blue HB stars, which at effective temperatures of about $8000 \mathrm{~K}$ to $20000 \mathrm{~K}$ show spectra rather similar (at moderate resolution) to main sequence stars of spectral types A and B and are therefore called HBA and HBB stars. In the field of the Milky Way such stars are often denominated by FHB (field HB star) and used as tracers for halo structure.

\section{Atmospheric parameters}

Already early studies of HBA and HBB stars in globular clusters showed discrepancies between observational results and theoretical expectations: Graham \& Doremus (1966) mentioned that the comparison of $\left(c_{1}\right)_{0}$ vs. $(b-y)_{0}$ for 50 blue HB stars in NGC 6397 with models from Mihalas (1966) indicated low surface gravities and a mean mass of $0.3 M_{\odot}\left(0.4 M_{\odot}\right)$ for solar (negligible) helium abundance, assuming $(\mathrm{m}-\mathrm{M})_{0}=12.0$ and $E_{\mathrm{B}-\mathrm{V}}=0.16$. Later spectroscopic analyses of $\mathrm{HB}$ stars (see cited papers for details) in globular clusters reproduced this effect (cf. Fig. 1): Crocker et al. (1988, M 3, M 5, M 15, M 92, NGC 288), de Boer et al. (1995, NGC 6397), Moehler et al. (1995, 1997a, M 15), and Moehler et al. (1997b, NGC 6752).

The zero age HB (ZAHB) in Fig. 1 marks the position where the HB stars have settled down and started to quietly burn helium in their cores. The terminal age HB

the decreasing sensitivity of $B-V$ to temperature on one hand and by the increasing Bolometric Correction for hotter stars (i.e., the maximum of stellar flux is radiated at ever shorter wavelengths for increasing temperatures, making stars fainter at $V$ ) on the other hand.

$\dagger$ Due to the higher opacities in their envelopes metal-rich HB stars are cooler than metal-poor ones with the same envelope mass. 


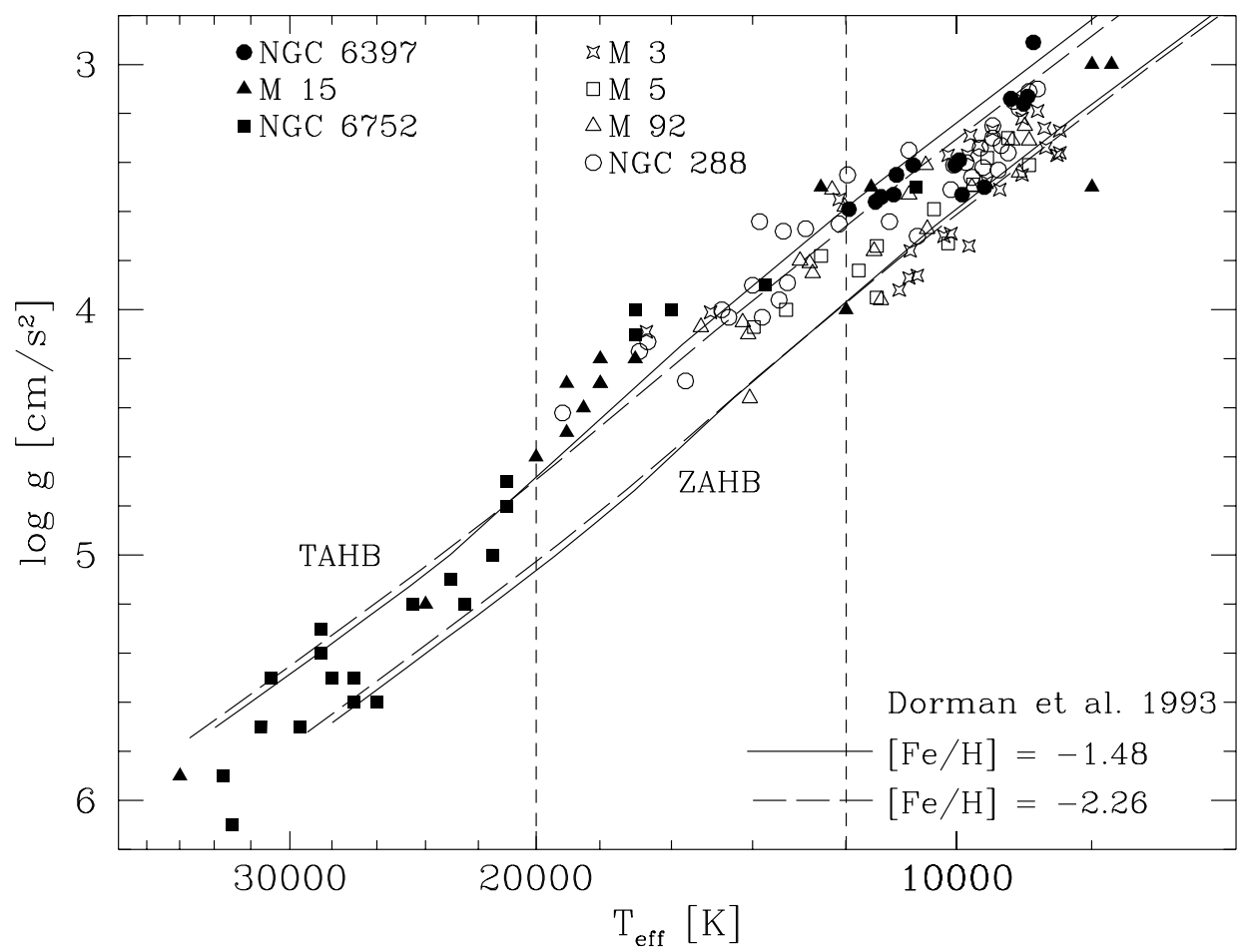

Figure 1. The results of Crocker et al. (1988, M 3, M 5, M 92, NGC 288), de Boer et al. (1995, NGC 6397), Moehler et al. (1995, 1997a, M 15), and Moehler et al. (1997b, NGC 6752) compared with evolutionary tracks from Dorman et al. (1993). ZAHB and TAHB stand respectively for the zero age and the terminal age HB (see text for details). The short-dashed lines mark the regions of $\operatorname{low} \log g$ (see text for details).

(TAHB) is defined by helium exhaustion in the core of the HB star $\left(Y_{\mathrm{C}}<0.0001\right)$. For temperatures between $12000 \mathrm{~K}$ and $20000 \mathrm{~K}$ the observed positions in the $\left(\log g, T_{\text {eff }}\right)$ diagram fall mostly above the ZAHB and in some cases even above the TAHB. This agrees with the finding of Saffer et al. (1997) that field HBB stars show a larger scatter away from the ZAHB in $T_{\text {eff }}, \log g$ than the hotter subdwarf B stars with $T_{\text {eff }}>20000 \mathrm{~K}$. Knowing the atmospheric parameters of the stars and the distances to the globular clusters we can determine masses for the stars (cf. Moehler et al. 1995, 1997b, de Boer et al. 1995). While the stars in M 3, M 5, and NGC 6752 have mean masses consistent with the canonical values, the hot HB stars in all other clusters have masses that are significantly lower than predicted by canonical HB evolution even for temperatures cooler than $12000 \mathrm{~K}$.

Also some UV observations suggest discrepancies between theoretical expectations and observational results: The IUE (International Ultraviolet Explorer) and HUT (Hopkins Ultraviolet Telescope) spectra of M 79 (Altner \& Matilsky 1993, Dixon et al. 1996) suggest lower than expected gravities and higher than expected metallicities for hot $\mathrm{HB}$ stars (but see Vink et al. 1999, who do not need low surface gravities to fit the HUT data). Hill et al. (1996) find from Ultraviolet Imaging Telescope (UIT) photometry of M 79 that stars bluer than $\mathrm{m}_{152}-\mathrm{m}_{249}=-0.2$ lie above the ZAHB, whereas cooler stars scatter around the ZAHB. UIT data of M 13 (Parise et al. 1998) find a lack of stars close to the ZAHB at a colour (temperature) range similar to the low $\log g$ range shown 
in Fig. 1. Landsman et al. (1996) on the other hand find good agreement between UIT photometry of blue stars in NGC 6752 and a standard ZAHB.

\section{Atmospheric abundances}

It has been realized early on that the blue HB and blue tail stars in globular clusters show weaker helium lines than field main sequence B stars of similar temperatures: Searle \& Rodgers (1966, NGC 6397); Greenstein \& Münch (1966, M 5, M 13, M 92); Sargent (1967, M 13, M 15, M 92). Greenstein et al. (1967) already suggested diffusion to explain this He deficiency. Michaud et al. (1983) performed the first theoretical study of diffusion effects in hot horizontal branch stars. Using the evolutionary tracks of Sweigart \& Gross (1976) they found for the metal-poor models that "in most of each envelope, the radiative acceleration on all elements (i.e., $\mathrm{C}, \mathrm{N}, \mathrm{O}, \mathrm{Ca}, \mathrm{Fe}$ ) is much larger than gravity which is not the case in main-sequence stars." The elements are thus pushed towards the surface of the star. Turbulence affects the different elements to varying extent, but generally reduces the overabundancest. Models without turbulence and/or mass loss (which may reduce the effects of diffusion) predict stronger He depletions than observed. A weak stellar wind could alleviate this discrepancy (Heber 1986, Michaud et al. 1989, Fontaine \& Chayer 1997, and Unglaub \& Bues 1998 discuss this effect, albeit for hotter stars). The extent of the predicted abundance variations varies with effective temperature, from none for HB stars cooler than about $5800 \pm 500 \mathrm{~K}$ (due to the very long diffusion timescales) to $2-4$ dex in the hotter stars (the hottest model has $T_{\text {eff }}=20700 \mathrm{~K}$ ) and also depends on the element considered.

Observations of blue HB and blue tail stars in globular clusters support the idea of diffusion being active above a certain temperature. Abundance analyses of blue HB stars cooler than $11000 \mathrm{~K}$ to $12000 \mathrm{~K}$ in general show no deviations from the globular cluster abundances derived from red giants, while for hotter stars departures from the general globular cluster abundances are found, e.g., iron enrichment to solar or even super-solar values and strong helium depletion (see Moehler 2001 for references and more details). This agrees with the finding of Altner \& Matilsky (1993) and Vink et al. (1999) that solar metallicity model atmospheres are required to fit the UV spectra of M 79.

All this evidence supports the suggestion of Grundahl et al. (1999) that the onset of diffusion in stellar atmospheres may play a role in explaining the jump along the $\mathrm{HB}$ towards brighter $u$ magnitudes at effective temperatures of about $11500 \mathrm{~K}$. This jump in $u, u-y$ is seen in all CMDs of globular clusters that have Strömgren photometry of sufficient quality. The effective temperature of the jump is roughly the same for all clusters, irrespective of metallicity, central density, concentration or mixing evidence, and coincides roughly with the onset of the "low $\log g$ problem" seen in Fig. 1 at $T_{\text {eff }} \approx$ $11000 \mathrm{~K}$ to $12000 \mathrm{~K}$. This in turn coincides with the region where surface convection zones due to hydrogen and He I ionization disappear in HB stars (Sweigart 2002).

Radiative levitation of heavy elements decreases the far-UV flux and by backwarming increases the flux in $u$. Grundahl et al. (1999) show that the use of metal-rich atmospheres $\left([\mathrm{Fe} / \mathrm{H}]=+0.5\right.$ for scaled-solar ATLAS9 Kurucz model atmospheres with $\log \epsilon_{\mathrm{Fe}}, \odot=$ 7.60) improves the agreement between observed data and theoretical ZAHB in the $u, u-y$-CMD at effective temperatures between $11500 \mathrm{~K}$ and $20000 \mathrm{~K}$, but it worsens

$\dagger$ Michaud (1982) and Charbonneau \& Michaud (1988) showed that meridional circulation can prevent gravitational settling and that the limiting rotational velocity decreases with decreasing $\log g$. Behr et al. (2000b) note that two of the HB stars hotter than $10000 \mathrm{~K}$ show higher rotational velocities and much smaller abundance deviations than other stars of similar temperature. 
the agreement between theory and observation for hotter stars in the Strömgren CMD of NGC 6752 (see their Fig. 8). Thus diffusion may either not be as important in the hotter stars or the effects may be diminished by a weak stellar wind. The gap at $(B-V)_{0} \approx 0$ discussed by Caloi (1999) is not directly related to the $u$-jump as it corresponds to an effective temperature of about $9000 \mathrm{~K}$ and is also not seen in every cluster (which would be expected if it were due to an atmospheric phenomenon). The gap at $T_{\text {eff }} \approx 13000 \mathrm{~K}$ seen in the $c_{1}, b-y$ diagram of field horizontal branch stars (Newell 1973, Newell \& Graham 1976) may be related to the $u$-jump as the $c_{1}$ index contains $u$.

The abundance distribution within a stellar atmosphere influences the temperature stratification and thereby the line profiles and the flux distribution of the emergent spectrum. A deviation in atmospheric abundances of HB stars from the cluster metallicity due to diffusion would thus affect their line profiles and flux distribution. Model atmospheres calculated for the cluster metallicity may then yield wrong results for effective temperatures and surface gravities when compared to observed spectra of HB stars. This effect could explain at least part of the observed discrepancies (see Sect. 5 for more details). Self-consistent model atmospheres taking into account the effects of gravitational settling and radiative levitation are, however, quite costly in CPU time and have started to appear only quite recently for hot stars (Dreizler \& Wolff 1999, Hui-Bon-Hoa et al. 2000).

\section{Rotational velocities}

Peterson (1983, 1985a, 1985b) found from high-resolution spectroscopic studies that clusters with bluer HB morphologies show higher rotation velocities among their HB stars. However, the analysis of Peterson et al. (1995) shows that while the stars in M 13 (which has a long blue tail) rotate on average faster than those in M 3 (which has only a short blue HB), the stars in NGC 288 and M 13 show slower rotation velocities at higher temperatures. These results are consistent with those reported by Behr et al. (2000a), who determined rotational velocities for stars as hot as $19000 \mathrm{~K}$ in M 13. They found that stars hotter than about $11000 \mathrm{~K}$ have significantly lower rotational velocities than cooler stars and that the change in mean rotational velocity may coincide with the gap seen along the blue HB of M 13 (see Ferraro et al. 1998 for an extensive discussion of gaps). Also the results of Cohen \& McCarthy (1997, M 92) and Behr et al. (2000b, M15) show that HB stars cooler than $\approx 11000 \mathrm{~K}$ to $12000 \mathrm{~K}$ in general rotate faster than hotter stars.

The studies by Behr (2003a) and Recio et al. (2002, 2004), which both consider several clusters, confirm the trends mentioned above. Below the diffusion threshold about $20 \%$ to $30 \%$ of the blue HB stars show rotation velocities of $v \sin i \approx 20 \ldots 30 \mathrm{~km} \mathrm{~s}^{-1}$, whereas there are no fast rotators among the hotter stars. From observations of field HB stars Behr (2003b) and Carney et al. (2003) note that red and cool blue HB stars show similar distributions of rotational velocities (after accounting for the larger radii of the red $\mathrm{HB}$ stars), whereas field RR Lyrae stars show no evidence for rotation.

Sills \& Pinsonneault (2000) studied theoretical models for the rotation of HB stars and find that the observed rotation of cool blue HB stars in M 13 can be explained if their red giant precursors have rapidly rotating cores and differential rotation in their convective envelopes and if angular momentum is redistributed from the rapidly rotating core to the envelope on the horizontal branch. If, however, turn-off stars rotate with less than $4 \mathrm{~km} \mathrm{~s}^{-1}$, rapidly rotating cores in the Main-Sequence stars (violating helioseismological results for the Sun) or an additional source of angular momentum on the red giant branch (e.g., mass transfer in close binaries or capture of a planet as described by Soker \& Harpaz 2000) are required to explain the rotation of blue HB stars. Talon \& Charbonnel (2004) 
speculate that such differential rotation could be understood within the framework of internal gravitational waves. The change in rotation rates towards higher temperatures is not predicted by the models of Sills \& Pinsonneault (2000) but could be understood as a result of gravitational settling of helium, which creates a mean molecular weight gradient, that then inhibits angular momentum transport to the surface of the star. Sweigart (2002) suggests that the weak stellar wind invoked to reconcile observed abundances in hot HB stars with diffusion calculations (cf. Sect. 3) could also carry away angular momentum from the surface layers and thus reduce the rotational velocities of these stars.

Soker \& Harpaz (2000) argue that the distribution of rotational velocities along the HB can be explained by the spin-up of their progenitors due to interactions with lowmass companions, predominantly gas-giant planets, in some cases also brown dwarfs or low-mass Main-Sequence stars (especially for the very hot HB stars). The slower rotation of the hotter stars in their scenario is explained by mass loss on the HB, which is accompanied by efficient angular momentum loss. This scenario, however, does not explain the sudden change in rotational velocities and the coincidence of this change with the onset of radiative levitation.

\section{Where do we stand?}

Analysis of a larger sample of hot HB stars in NGC 6752 (Moehler et al. 2000) showed that the use of model atmospheres with solar or super-solar abundances removes much of the deviation from canonical tracks in effective temperature, surface gravity and mass for hot $\mathrm{HB}$ stars discussed in Sect. 2. However, some discrepancies remain, indicating that the low $\log g$, low mass problem cannot be completely solved by scaled-solar metalrich atmospheres (which do reproduce the $u$-jump reported by Grundahl et al. 1999). As Michaud et al. (1983) noted diffusion will not necessarily enhance all heavy elements by the same amount and the effects of diffusion vary with effective temperature. Elements that were originally very rare may be enhanced even stronger than iron. The question of whether diffusion can fully explain the "low gravity" problem cannot be answered without detailed abundance analyses to determine the actual abundances and subsequent analyses using model atmospheres with non-scaled solar abundances (like ATLAS12, Kurucz 1992). Additional caution is also recommended by the results of Moehler et al. (2003) on M 13, where even the use of the metal-rich model atmospheres does not eliminate the problem of the low masses or the low gravities for effective temperatures between $12000 \mathrm{~K}$ and $16000 \mathrm{~K}$. In that cluster we also found significant disagreement between atmospheric parameters derived from Strömgren photometry and from Balmer line fitting for stars cooler than about $9000 \mathrm{~K}$, for which we found no explanation.

Still unexplained are also the low masses found for cool blue HB stars (which are not affected by diffusion) in, e.g., NGC 6397 and M 92. For those stars a longer distance scale to globular clusters would reduce the discrepancies (Moehler 1999). Such a longer distance scale has been suggested by several authors using HIPPARCOS results for metal-poor field subdwarfs to determine the distances to globular clusters by fitting their main sequence with the local subdwarfs. One should note, however, that de Boer et al. (1997), report that Hipparcos parallaxes for field HBA stars still yield masses significantly below the canonical mass expected for these objects. Carretta et al. (2000) present an extensive and excellent discussion of various globular cluster distance determinations and the zoo of biases that affect them, while Gratton et al. (2003) concentrate on the error budget of distances from main sequence fitting in their paper.

The problem of the different rotation velocities for cool and hot HB stars, however, remains rather stubbornly opposed to any attempted solution. 


\section{Acknowledgements}

I would like to thank my colleagues and collaborators Drs. A.V. Sweigart, W.B. Landsman, M. Lemke, U. Heber, and B.B. Behr for many fruitful discussions and helpful comments.

\section{References}

Altner B., Matilsky T.A., 1993, ApJ 410, 116

Behr, B.B. 2003a, ApJS 149, 67

Behr, B.B. 2003b, ApJS 149, 101

Behr B.B., Djorgovski S.G., Cohen J.G., et al. , 2000a, ApJ 528, 849

Behr B.B., Cohen J.G., McCarthy J.K., 2000b, ApJ 531, L37

Caloi V., 1999, A\&A 343, 904

Carney, B.W., Latham, D.W., Stefanik, R.P., Laird, J.B., Morse, J.A. 2003, AJ 125, 293

Carretta E., Gratton R., Clementini G., Fusi Pecci F., 2000, ApJ 533, 215

Charbonneau P., Michaud G., 1988, ApJ 327, 809

Cohen J.G., McCarthy J.K., 1997, AJ 113, 1353

Crocker D.A., Rood R.T., O'Connell R.W., 1988, ApJ 332, 236

de Boer K.S., Schmidt J.H.K., Heber U., 1995, A\&A 303, 95

de Boer K.S., Tucholke H.-J., Schmidt J.H.K., 1997, A\&A 317, L23

Dixon W.V., Davidsen A.F., Dorman B., Ferguson H.C., 1996, AJ 111, 1936

Dorman B., Rood, R.T., O'Connell, W.O., 1993, ApJ 419, 596

Dreizler S., Wolff B., 1999, A\&A 348, 189

Faulkner J., 1966, ApJ 144, 978

Ferraro F.R., Paltrinieri B., Fusi Pecci F., Dorman B., Rood R.T., 1998, ApJ 500, 311

Fontaine G., Chayer P., 1997, in The $3^{\text {rd }}$ Conf. on Faint Blue Stars, eds. A.G.D. Philip, J. Liebert \& R.A. Saffer (L. Davis Press, Schenectady), p. 169

Graham J.A., Doremus C., 1966, AJ 73, 226

Gratton R.G., Bragaglia A., Carretta E., Clementini G., Desidera S., Grundahl F., Lucatello S., 2003, A\&A 408, 529

Greenstein G.S., Münch G., 1966, ApJ 146, 518

Greenstein G.S., Truran J.W., Cameron A.G.W., 1967, Nature 213, 871

Grundahl F., Catelan M., Landsman W.B., Stetson P.B., Andersen M., 1999, ApJ 524, 242

Heber U., 1986, A\&A 155, 33

Hill R.S., Cheng K.-P., Smith E.P., et al. , 1996, AJ 112, 601

Hoyle F., Schwarzschild M., 1955, ApJS 2, 1

Hui-Bon-Hoa A., LeBlanc F., Hauschildt P., 2000, ApJ 535, L43

Iben I.Jr., Rood R.T., 1970, ApJ 161, 587

Kurucz R.L., 1992, in The Stellar Populations of Galaxies, eds. B. Barbuy \& A. Renzini, IAU Symp. 149 (Kluwer:Dordrecht), 225

Landsman W.B., Sweigart A.V., Bohlin R.C., et al. , 1996, ApJ 472, L93

Michaud G., 1982, ApJ 258, 349

Michaud G., Vauclair G., Vauclair S., 1983, ApJ 267, 256

Michaud G., Bergeron P., Heber U., Wesemael F., 1989, ApJ 338, 417

Mihalas D.M., 1966, ApJS 13, 1

Moehler S., 1999, Reviews in Modern Astronomy, ed. R. Schielicke, Vol. 12, p. 281

Moehler S., 2001, PASP 113, 1162

Moehler S., Heber U., de Boer K.S., 1995, A\&A 294, 65

Moehler S., Heber U., Durrell P., 1997a, A\&A 317, L83

Moehler S., Heber U., Rupprecht G., 1997b, A\&A 319, 109

Moehler S., Sweigart A.V., Landsman W., Heber U., 2000, A\&A 360, 120

Moehler S., Landsman W.B., Sweigart A.V., Grundahl F., 2003, A\&A 405, 135

Newell E.B., 1973, ApJS 26, 37

Newell E.B., Graham J.A., 1976, ApJ 204, 804 
Parise R.A., Bohlin R.C., Neff S.G., et al. , 1998, ApJ 501, L67

Peterson R.C., 1983, ApJ 275, 737

Peterson R.C., 1985a, ApJ 289, 320

Peterson R.C., 1985b, ApJ 294, L35

Peterson R.C., Rood R.T., Crocker D.A., 1995, ApJ 453, 214

Recio-Blanco, A., Piotto, G., Aparicio, A., Renzini, A. 2002, ApJ 527, L71

Recio-Blanco, A., Piotto, G., Aparicio, A., Renzini, A. 2004, A\&A 417, 597

Rood R.T., 1973, ApJ 184, 815

Saffer R.A., Keenan F.P., Hambly N.C., Dufton P.L., Liebert J., 1997, ApJ 491, 172

Sandage A.R., Wallerstein G., 1960, ApJ 131, 598

Sargent W.L.W., 1967, ApJ 148, L147

Searle L., Rodgers A.W., 1966, ApJ 143, 809

Shapley H., 1915, Contr. Mt. Wilson 116

Sills A., Pinsonneault M.H., 2000, ApJ 540, 489

Soker N., Harpaz A., 2000, MNRAS 317, 861

Sweigart A.V., 1994, in Hot Stars in the Galactic Halo, eds. S. Adelman, A. Upgren, C.J. Adelman, (Cambridge University Press, Cambridge), p. 17

Sweigart A.V., 2002, Highlights of Astronomy, Vol. 12, ed. H. Rickman (ASP), p. 292

Sweigart A.V., Gross P.G., 1976, ApJS 32, 367

Talon S., Charbonnel C., 2004, A\&A 418, 1051

ten Bruggencate P., 1927, Sternhaufen (Julius Springer Verlag, Berlin)

Unglaub K., Bues I., 1998, A\&A 338, 75

Vink J.S., Heap S.R., Sweigart A.V., Lanz T., Hubeny I., 1999, A\&A 345, 109

\section{Discussion}

FREYTAG: What are "real" abundances in contrast to "diffusion" abundances? Can they still be reliably defined?

MOEHLER: By real abundances I mean the abundances, which the star would show if its atmosphere were not affected by diffusion (like the abundances derived from red giants in globular clusters). Those cannot be derived if the spectrum is affected by diffusion

Preston: Have you compared the abundances of hot HB stars with those derived from red giants, which are affected in a different way by diffusion processes?

MoeHLeR: Red giants should show no diffusion effects in their atmospheres due to their deep convection zones. HB stars with diffusion usually show strong enhancements of heavy elements compared to red giants in the same cluster.

Corbally: Some dozen years ago I was working with Richard Gray on the field horizontal branch stars identified by Dave Philip. These were all slightly cooler than those you have shown, i.e., truly A-type horizontal branch stars. We found that they lay near or slightly above the Main Sequence, indeed they were metal-poor as you said, and that also from their Balmer line profiles at medium resolution were probably helium-rich. Could you comment on the last possibility?

MoEHLer: Not really, as I find this a very puzzling result, which cannot be easily understood in the terms of diffusion. 